\title{
The diagonal of a multicosimplicial object
}

\author{
Philip S. Hirschhorn ${ }^{1}$
}

Received: 16 September 2015 / Accepted: 14 February 2017 / Published online: 20 March 2017

(C) Tbilisi Centre for Mathematical Sciences 2017

\begin{abstract}
We show that the functor that takes a multicosimplicial object in a model category to its diagonal cosimplicial object is a right Quillen functor. This implies that the diagonal of a Reedy fibrant multicosimplicial object is a Reedy fibrant cosimplicial object, which has applications to the calculus of functors. We also show that, although the diagonal functor is a Quillen functor, it is not a Quillen equivalence for multicosimplicial spaces. We also discuss total objects and homotopy limits of multicosimplicial objects. We show that the total object of a multicosimplicial object is isomorphic to the total object of the diagonal, and that the diagonal embedding of the cosimplicial indexing category into the multicosimplicial indexing category is homotopy left cofinal, which implies that the homotopy limits are weakly equivalent if the multicosimplicial object is at least objectwise fibrant.
\end{abstract}

Keywords Multicosimplicial · Cosimplicial · Diagonal · Total space · Totalization · Quillen functor

Mathematics Subject Classification 55U35 - 18G55

\section{Contents}

1 Introduction . . . . . . . . . . . . . . . . . . . . . . . . 972

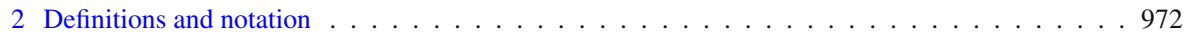

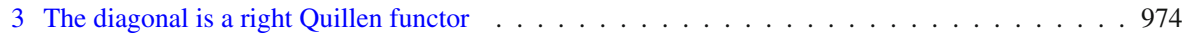

Communicated by Haynes Miller.

$\bowtie$ Philip S. Hirschhorn

psh@math.mit.edu

1 Department of Mathematics, Wellesley College, Wellesley, MA 02481, USA 


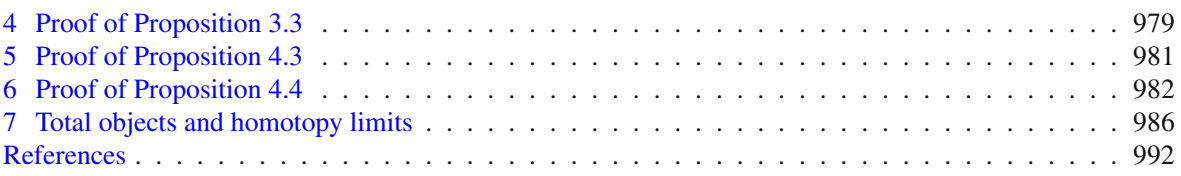

\section{Introduction}

After constructing a multicosimplicial object, it is common to pass to its diagonal cosimplicial object. In order for the total object of the diagonal to have homotopy meaning, though, you need to know that that diagonal is fibrant.

We prove in Theorem 3.6 that the functor that takes a multicosimplicial object in a model category to its diagonal cosimplicial object is a right Quillen functor. This implies that the diagonal of a fibrant multicosimplicial object is a fibrant cosimplicial object, which has applications to the calculus of functors (see [1]). We also show in Theorem 3.12 and Corollary 3.13 that, although the diagonal functor is a Quillen functor, it is not a Quillen equivalence for multicosimplicial spaces.

In Sect. 7 we discuss total objects and homotopy limits. We show in Theorem 7.5 that the total object of a multicosimplicial object is isomorphic to the total object of the diagonal, and in Proposition 7.11 that the diagonal embedding of the cosimplicial indexing category into the multicosimplicial indexing category is homotopy left cofinal, which implies that the homotopy limits are weakly equivalent if the multicosimplicial object is at least objectwise fibrant (see Theorem 7.12). We also show in Theorem 7.10 that the total object of a Reedy fibrant multicosimplicial object has a natural weak equivalence to the homotopy limit, and that this natural weak equivalence interacts well with the isomorphism between the total object of the multicosimplicial object and the total object of its diagonal (see Theorem 7.13).

\section{Definitions and notation}

Notation 2.1 If $n$ is a nonnegative integer, we let $[n]$ denote the ordered set $(0,1,2, \ldots, n)$. We will use $\boldsymbol{\Delta}$ to denote the cosimplicial indexing category, which is the category with objects the $[n]$ for $n \geq 0$ and with morphisms $\boldsymbol{\Delta}([n],[k])$ the weakly monotone functions $[n] \rightarrow[k]$.

Definition 2.2 If $\mathcal{M}$ is a category, a cosimplicial object in $\mathcal{M}$ is a functor $\Delta \rightarrow \mathcal{M}$, and the category of cosimplicial objects in $\mathcal{M}$ is the functor category $\mathcal{M}^{\boldsymbol{\Delta}}$. If $\boldsymbol{X}$ is a cosimplicial object in $\mathcal{M}$, then we will generally denote the value of $\boldsymbol{X}$ on $[k]$ as $\boldsymbol{X}^{k}$.

Notation 2.3 If $n$ is a positive integer, then we will let $\Delta^{n}$ denote the product category $\underbrace{\Delta \times \Delta \times \cdots \times \Delta}_{n \text { times }}$.

Definition 2.4 If $\mathcal{M}$ is a category and $n$ is a positive integer, then an $n$-cosimplicial object in $\mathcal{M}$ is a functor $\boldsymbol{\Delta}^{n} \rightarrow \mathcal{M}$. If $\boldsymbol{X}$ is an $n$-cosimplicial object in $\mathcal{M}$, then we will generally denote the value of $\boldsymbol{X}$ on $\left(\left[k_{1}\right],\left[k_{2}\right], \ldots,\left[k_{n}\right]\right)$ by $\boldsymbol{X}^{\left(k_{1}, k_{2}, \ldots, k_{n}\right)}$. 


\subsection{The diagonal}

An $n$-cosimplicial object in a category $\mathcal{M}$ is a functor $\boldsymbol{X}: \boldsymbol{\Delta}^{n} \rightarrow \mathcal{M}$, and we can restrict that functor to the "diagonal subcategory" of $\boldsymbol{\Delta}^{n}$ to obtain a cosimplicial object diag $\boldsymbol{X}$ in $\mathcal{M}$.

Definition 2.5 Let $n$ be a positive integer.

1. The diagonal embedding of the category $\boldsymbol{\Delta}$ into $\boldsymbol{\Delta}^{n}$ is the functor $D: \boldsymbol{\Delta} \rightarrow \boldsymbol{\Delta}^{n}$ that takes the object $[k]$ of $\boldsymbol{\Delta}$ to the object $(\underbrace{[k],[k], \ldots,[k]})$ of $\boldsymbol{\Delta}^{n}$ and the $n$ times morphism $\phi:[p] \rightarrow[q]$ of $\boldsymbol{\Delta}$ to the morphism $\phi^{n}:(\underbrace{[p],[p], \ldots,[p]}_{n \text { times }}) \rightarrow$ $(\underbrace{[q],[q], \ldots,[q]}_{n \text { times }})$ of $\boldsymbol{\Delta}^{n}$.

2. If $\mathcal{M}$ is a category and $\boldsymbol{X}$ is an $n$-cosimplicial object in $\mathcal{M}$, then the diagonal diag $\boldsymbol{X}$ of $\boldsymbol{X}$ is the cosimplicial object in $\mathcal{M}$ that is the composition $\Delta \stackrel{D}{\rightarrow} \Delta^{n} \stackrel{\boldsymbol{X}}{\rightarrow} \mathcal{M}$, so that $(\operatorname{diag} \boldsymbol{X})^{k}=\boldsymbol{X}^{(k, k, \ldots, k)}$.

\subsection{Matching objects and latching objects}

If $\mathcal{C}$ is a Reedy category (see [6, Def. 15.1.2]), $\mathcal{M}$ is a model category, $\boldsymbol{X}$ is a $\mathcal{C}$-diagram in $\mathcal{M}$, and $\alpha$ is an object of $\mathcal{C}$, then we will use the notation of [6, Def. 15.2.5] and denote the matching object of $\boldsymbol{X}$ at $\alpha$ by $\mathrm{M}_{\alpha} \boldsymbol{X}$, or by $\mathrm{M}_{\alpha}^{\mathrm{C}} \boldsymbol{X}$ if the indexing category isn't obvious.

Note that in the case of cosimplicial objects, our notation for matching objects (see [6, Def. 15.2.5]) differs from that of [4, Ch. X, §4], in that we index a matching object by the degree at which it is the matching object, whereas [4, Ch. X, §4] indexes it by one less than that. Thus, our notation for the matching map of a cosimplicial object $\boldsymbol{X}$ at $[k]$ is $\boldsymbol{X}^{k} \rightarrow \mathrm{M}_{k} \boldsymbol{X}$, while the notation of [4, Ch. X, §4] is $\boldsymbol{X}^{k} \rightarrow \mathrm{M}^{k-1} \boldsymbol{X}$.

Definition 2.6 Let $\mathcal{C}$ be a Reedy category, let $\mathcal{M}$ be a model category, let $\boldsymbol{X}$ be a $\mathcal{C}$-diagram in $\mathcal{M}$, and let $\alpha$ be an object of $\mathcal{C}$.

1. The matching category $\partial(\alpha \downarrow \overleftarrow{\mathcal{C}})$ of $\mathcal{C}$ at $\alpha$ is the full subcategory of $(\alpha \downarrow \overleftarrow{\mathcal{C}})$ (the category with objects the weakly degree-lowering maps with domain $\alpha$ and with morphisms the commutative triangles; see [6, Def. 7.6.1]) containing all of the objects except the identity map of $\alpha$ (see [6, Def. 15.2.3]).

2. The matching object of $\boldsymbol{X}$ at $\alpha$ is $\mathrm{M}_{\alpha} \boldsymbol{X}=\lim _{\partial(\alpha \downarrow \overleftarrow{\mathrm{e}})} \boldsymbol{X}$ and the matching map of $\boldsymbol{X}$ at $\alpha$ is the natural map $\boldsymbol{X}_{\alpha} \rightarrow \mathrm{M}_{\alpha} \boldsymbol{X}$. We will use $\mathrm{M}_{\alpha}^{\mathrm{C}} \boldsymbol{X}$ to denote the matching object if the indexing category isn't obvious.

3. The latching category $\partial(\overrightarrow{\mathrm{e}} \downarrow \alpha)$ of $\mathcal{C}$ at $\alpha$ is the full subcategory of ( $\vec{e} \downarrow \alpha$ ) (the category with objects the weakly degree-raising maps with codomain $\alpha$ and with morphisms the commutative triangles; see [6, Def. 7.6.1]) containing all of the objects except the identity map of $\alpha$ (see [6, Def. 15.2.3]). 


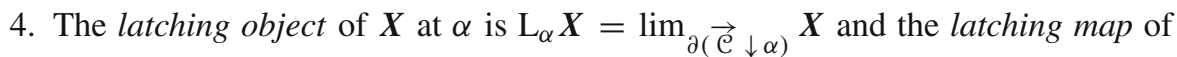
$\boldsymbol{X}$ at $\alpha$ is the natural map $\mathrm{L}_{\alpha} \boldsymbol{X} \rightarrow \boldsymbol{X}_{\alpha}$. We will use $\mathrm{L}_{\alpha}^{\mathrm{C}} \boldsymbol{X}$ to denote the latching object if the indexing category isn't obvious.

Definition 2.7 [6, Def. 15.3.3] Let $\mathcal{C}$ be a Reedy category and let $\mathcal{M}$ be a model category. A map $f: \boldsymbol{X} \rightarrow \boldsymbol{Y}$ of $\mathcal{C}$-diagrams in $\mathcal{M}$ is

- a Reedy fibration if for every object $\alpha$ of $\mathcal{C}$ the relative matching map $\boldsymbol{X}_{\alpha} \rightarrow$ $\boldsymbol{Y}_{\alpha} \times \mathrm{M}_{\alpha} \boldsymbol{Y} \mathbf{M}_{\alpha} \boldsymbol{X}$ is a fibration in $\mathcal{M}$ (see, e.g., Definition 3.1),

- a Reedy cofibration if for every object $\alpha$ of $\mathcal{C}$ the relative latching map $\boldsymbol{X}_{\alpha} \amalg_{\mathrm{L}_{\alpha} \boldsymbol{X}}$ $\mathrm{L}_{\alpha} \boldsymbol{Y} \rightarrow \boldsymbol{Y}_{\alpha}$ is a cofibration in $\mathcal{M}$, and

- a Reedy weak equivalence if for every object $\alpha$ of $\mathcal{C}$ the map $\boldsymbol{X}_{\alpha} \rightarrow \boldsymbol{Y}_{\alpha}$ is a weak equivalence in $\mathcal{M}$.

Thus,

- an object $\boldsymbol{X}$ of $\mathcal{M}$ is Reedy fibrant if for every object $\alpha$ of $\mathcal{C}$ the matching map $\boldsymbol{X}_{\alpha} \rightarrow \mathrm{M}_{\alpha} \boldsymbol{X}$ is a fibration in $\mathcal{M}$, and

- an object $\boldsymbol{X}$ of $\mathcal{M}$ is Reedy cofibrant if for every object $\alpha$ of $\mathcal{C}$ the latching map $\mathrm{L}_{\alpha} \boldsymbol{X} \rightarrow \boldsymbol{X}_{\alpha}$ is a cofibration in $\mathcal{M}$.

\section{The diagonal is a right Quillen functor}

We prove in Theorem 3.6 that the functor that takes a multicosimplicial object to its diagonal cosimplicial object is a right Quillen functor.

Definition 3.1 Let $\mathcal{M}$ be a model category, let $n$ be a positive integer, and let $\boldsymbol{X} \rightarrow \boldsymbol{Y}$ be a map of $n$-cosimplicial objects in $\mathcal{M}$. For every nonnegative integer $k$, the matching objects of $\boldsymbol{X}$ in $\mathcal{M}^{\boldsymbol{\Delta}^{n}}$ at $([k],[k], \ldots,[k])$ and of $\operatorname{diag} \boldsymbol{X}$ in $\mathcal{M}^{\boldsymbol{\Delta}}$ at $[k]$ are

$$
\mathbf{M}_{([k],[k], \ldots,[k])}^{\boldsymbol{\Delta}^{n}} \boldsymbol{X}=\lim _{\partial\left(([k],[k], \ldots,[k]) \downarrow \overleftarrow{\Delta^{n}}\right)} \boldsymbol{X} \quad \text { and } \quad \mathbf{M}_{[k]}^{\Delta} \operatorname{diag} \boldsymbol{X}=\lim _{\partial([k] \downarrow \overleftarrow{\Delta})} \operatorname{diag} \boldsymbol{X}
$$

(with similar formulas for $\boldsymbol{Y}$ ), and we define $P_{k}^{\boldsymbol{\Delta}^{n}}$ and $P_{k}^{\boldsymbol{\Delta}}$ by letting the following diagrams be pullbacks:
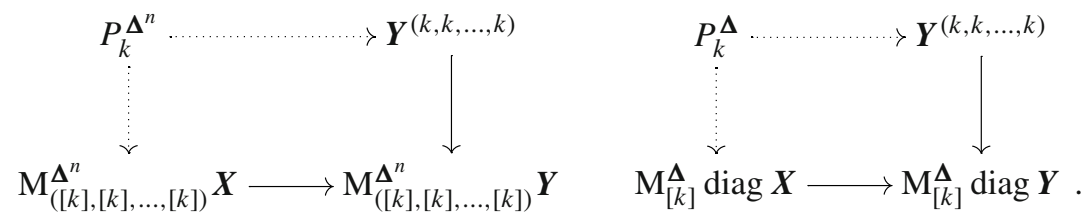

Thus,

- if the map $\boldsymbol{X} \rightarrow \boldsymbol{Y}$ is a Reedy fibration of $n$-cosimplicial objects then the natural map $\boldsymbol{X}^{(k, k, \ldots, k)} \rightarrow P_{k}^{\boldsymbol{\Delta}^{n}}$ (being among the the relative matching maps) is a fibration for all $k \geq 0$, and 
- the map diag $\boldsymbol{X} \rightarrow \operatorname{diag} \boldsymbol{Y}$ is a Reedy fibration of cosimplicial objects if and only if the natural map $\boldsymbol{X}^{(k, k, \ldots, k)} \rightarrow P_{k}^{\Delta}$ is a fibration for all $k \geq 0$

(see Definition 2.7). Since we are viewing $\Delta$ as a subcategory of $\Delta^{n}$, there are natural maps

$$
\begin{aligned}
& \mathbf{M}_{([k],[k], \ldots,[k])}^{\boldsymbol{\Delta}^{n}} \boldsymbol{X}=\lim _{\partial\left(([k],[k], \ldots,[k]) \downarrow \overleftarrow{\boldsymbol{\Delta}^{n}}\right)} \boldsymbol{X} \longrightarrow \lim _{\partial([k] \downarrow \overleftarrow{\boldsymbol{\Delta}})} \operatorname{diag} \boldsymbol{X}=\mathbf{M}_{[k]}^{\boldsymbol{\Delta}} \operatorname{diag} \boldsymbol{X} \quad \text { and } \\
& \mathbf{M}_{([k],[k], \ldots,[k])}^{\boldsymbol{\Delta}^{n}} \boldsymbol{Y}=\lim _{\partial\left(([k],[k], \ldots,[k]) \downarrow \overleftarrow{\boldsymbol{\Delta}^{n}}\right)} \boldsymbol{Y} \longrightarrow \lim _{\partial([k] \downarrow \overleftarrow{\boldsymbol{\Delta}})} \operatorname{diag} \boldsymbol{Y}=\mathbf{M}_{[k]}^{\boldsymbol{\Delta}} \operatorname{diag} \boldsymbol{Y}
\end{aligned}
$$

and those induce a natural map

$$
P_{k}^{\Delta^{n}} \longrightarrow P_{k}^{\Delta}
$$

Proposition 3.3 If $\mathcal{M}$ is a model category, $n$ is a positive integer, and $\boldsymbol{X} \rightarrow \boldsymbol{Y}$ is a Reedy fibration of $n$-cosimplicial objects in $\mathcal{M}$, then the natural map (3.2) is a fibration.

The proof of Proposition 3.3 is in Sect. 4; it depends on results that are proved in Sects. 5 and 6.

Theorem 3.4 If $\mathcal{M}$ is a model category, $n$ is a positive integer, and $\boldsymbol{X} \rightarrow \boldsymbol{Y}$ is a Reedy fibration of $n$-cosimplicial objects in $\mathcal{M}$, then the induced map of diagonals

$$
\operatorname{diag} \boldsymbol{X} \longrightarrow \operatorname{diag} \boldsymbol{Y}
$$

is a Reedy fibration of cosimplicial objects.

Proof We must show that for every nonnegative integer $k$ the map

$$
(\operatorname{diag} \boldsymbol{X})^{k}=\boldsymbol{X}^{(k, k, \ldots, k)} \longrightarrow P_{k}^{\Delta}
$$

(see Definition 3.1) is a fibration. That map is the composition

$$
\boldsymbol{X}^{(k, k, \ldots, k)} \longrightarrow P_{k}^{\boldsymbol{\Delta}^{n}} \longrightarrow P_{k}^{\Delta}
$$

The first of those is a fibration because the map $\boldsymbol{X} \rightarrow \boldsymbol{Y}$ is a Reedy fibration of $n$-cosimplicial objects, and Proposition 3.3 is the statement that the second is also a fibration.

Special cases of the following corollary are already known (see [9, Lem. 7.1], in view of [5, Prop. 5.8]).

Corollary 3.5 If $\mathcal{M}$ is a model category, $n$ is a positive integer, and $\boldsymbol{X}$ is a Reedy fibrant $n$-cosimplicial object in $\mathcal{M}$, then the diagonal cosimplicial object $\operatorname{diag} \boldsymbol{X}$ is Reedy fibrant.

Proof This follows from Theorem 3.4 by letting $\boldsymbol{Y}$ be the constant $n$-cosimplicial object at the terminal object of $\mathcal{M}$. 
Theorem 3.6 If $\mathcal{M}$ is a model category and $n$ is a positive integer, then the diagonal functor diag: $\mathcal{N}^{\Delta^{n}} \rightarrow \mathcal{M}^{\Delta}$, which takes an n-cosimplicial object in $\mathcal{N}$ to its diagonal cosimplicial object, is a right Quillen functor (see [6, Def. 8.5.2]).

Proof Since a model category is cocomplete, the left Kan extension of a cosimplicial object along the diagonal inclusion $\boldsymbol{\Delta} \rightarrow \boldsymbol{\Delta}^{n}$ always exists (see [2, Thm. 3.7.2] or [8, Thm. 17.1.6] or, for the dual statement, [7, Cor. X.3.2]), and so the diagonal functor has a left adjoint. Thus, we need only show that the diagonal functor preserves both fibrations and trivial fibrations (see [6, Prop. 8.5.3]).

Theorem 3.4 implies that that the diagonal preserves fibrations. Since the weak equivalences of cosimplicial objects and of $n$-cosimplicial objects are defined degreewise, the restriction of an $n$-cosimplicial object to its diagonal preserves all weak equivalences, and so it also preserves trivial fibrations.

\subsection{The multicosimplicial product of standard simplices}

The main result of this section is Theorem 3.11, which we will use in the following section to show that the right Quillen functor of Theorem 3.6 is not a Quillen equivalence. It will also be used in Theorem 7.5 to show that the total object of a multicosimplicial object is isomorphic to the total object of its diagonal cosimplicial object.

Definition 3.7 If $F: \mathcal{A} \rightarrow \mathcal{B}$ is a functor between small categories and $X$ is an object of $\mathcal{B}$, then $\mathcal{B}(F-, X)$ is the $\mathcal{A}^{\text {op }}$-diagram of sets that on an object $W$ of $\mathcal{A}$ is the set $\mathcal{B}(F W, X)$. This is natural in $X$, and thus defines a $\mathcal{B}$-diagram of $\mathcal{A}^{\text {op }}$-diagrams of sets.

If $F: \mathcal{A} \rightarrow \mathcal{B}$ is the identity functor of $\boldsymbol{\Delta}$, then an $\mathcal{A}^{\mathrm{op}}$-diagram of sets is a $\boldsymbol{\Delta}^{\mathrm{op}}{ }_{-}$ diagram of sets, i.e., a simplicial set, and so this defines a cosimplicial object in the category of simplicial sets, i.e., a cosimplicial simplicial set, which we will denote by $\Delta$. If $k$ is a nonnegative integer, then for a nonnegative integer $i$ the $i$-simplices of $\boldsymbol{\Delta}(-,[k])$ are the maps $\boldsymbol{\Delta}([i],[k])$, i.e., the weakly monotone functions $[i] \rightarrow[k]$, and so the simplicial set $\boldsymbol{\Delta}(-,[k])$ is the standard $k$-simplex, which we will denote by $\Delta[k]$. That is, $\Delta: \Delta \rightarrow$ SS is a cosimplicial simplicial set, and its value on the object $[k]$ is $\Delta[k]$. We will call it the cosimplicial standard simplex.

If $n$ is a positive integer and $F: \mathcal{A} \rightarrow \mathcal{B}$ is the diagonal embedding $D: \Delta \rightarrow \boldsymbol{\Delta}^{n}$ (see Definition 2.5), then this defines a $\Delta^{n}$-diagram of simplicial sets, i.e., an $n$ cosimplicial simplicial set, which we will denote by $\Delta^{(n)}$. If $\left(\left[p_{1}\right],\left[p_{2}\right], \ldots,\left[p_{n}\right]\right)$ is an object of $\boldsymbol{\Delta}^{n}$, then the simplicial set $\boldsymbol{\Delta}^{n}\left(D-,\left(\left[p_{1}\right],\left[p_{2}\right], \ldots,\left[p_{n}\right]\right)\right)$ has as its $k$-simplices the $n$-tuples of maps

$$
\left(\alpha_{1}, \alpha_{2}, \ldots, \alpha_{n}\right):([k],[k], \ldots,[k]) \longrightarrow\left(\left[p_{1}\right],\left[p_{2}\right], \ldots,\left[p_{n}\right]\right)
$$

where each $\alpha_{i}:[k] \rightarrow\left[p_{i}\right]$ is a weakly monotone map. Thus, each $k$-simplex is the product for $1 \leq i \leq n$ of a $k$-simplex of $\Delta\left[p_{i}\right]$, i.e., a $k$-simplex of $\Delta\left[p_{1}\right] \times \Delta\left[p_{2}\right] \times$ $\cdots \times \Delta\left[p_{n}\right]$, and so $\Delta^{(n)}\left(\left[p_{1}\right],\left[p_{2}\right], \ldots,\left[p_{n}\right]\right)$ is the product of standard simplices $\Delta\left[p_{1}\right] \times \Delta\left[p_{2}\right] \times \cdots \times \Delta\left[p_{n}\right]$. That is, $\Delta^{(n)}: \Delta^{n} \rightarrow$ SS is an $n$-cosimplicial simplicial 
set whose value on the object $\left(\left[p_{1}\right],\left[p_{2}\right], \ldots,\left[p_{n}\right]\right)$ is $\Delta\left[p_{1}\right] \times \Delta\left[p_{2}\right] \times \cdots \times \Delta\left[p_{n}\right]$. We will call it the $n$-cosimplicial product of standard simplices.

Lemma 3.8 If $n$ is a positive integer, then the $n$-cosimplicial product of standard simplices $\Delta^{(n)}$ (see Definition 3.7) is a Reedy cofibrant (see [6, Def. 15.3.3])n-cosimplicial simplicial set.

Proof The latching map at the object $\left(\left[p_{1}\right],\left[p_{2}\right], \ldots\left[p_{n}\right]\right)$ of $\Delta^{n}$ is the inclusion of the boundary of $\Delta\left[p_{1}\right] \times \Delta\left[p_{2}\right] \times \cdots \times \Delta\left[p_{n}\right]$, and is thus a cofibration.

Definition 3.9 If $K$ is a simplicial set, then the category of simplices $\Delta K$ of $K$ is the category that has as objects the simplices of $K$ and as morphisms from $\sigma$ to $\tau$ the simplicial operators that take $\tau$ to $\sigma$ (see [6, Def. 15.1.16]).

Lemma 3.10 If $K$ is a simplicial set and $\Delta K$ is the category of simplices of $K$ (see Definition 3.9), then $K$ is naturally isomorphic to the colimit of the $\Delta K$-diagram of simplicial sets that

- takes an n-simplex of $K$ to the standard $n$-simplex $\Delta[k]$,

- when $\partial_{i}(\tau)=\sigma$, takes $\partial_{i}$ to the inclusion of the image of $\sigma$ as the $i$ 'th face of the image of $\tau$, and

- when $s^{i}(\tau)=\sigma$, takes $s^{i}$ to the collapse of the image of $\sigma$ to the image of $\tau$ that identifies vertices $i$ and $i+1$

under an isomorphism that for an $n$-simplex $\sigma$ takes the nondegenerate $n$-simplex of $\Delta[n]$ to $\sigma$.

Proof See [6, Prop. 15.1.20].

The following theorem is the degree 0 part of [5, Remark on p. 172] and [9, Prop. 8.1]. The full statement, for a general model category, is Theorem 7.5.

Theorem 3.11 Let $n$ be a positive integer.

1. The left Kan extension of the cosimplicial standard simplex $\Delta$ (see Definition 3.7) along the diagonal embedding $\boldsymbol{\Delta} \rightarrow \boldsymbol{\Delta}^{n}$ (see Definition 2.5) is the $n$ cosimplicial product of standard simplices $\Delta^{(n)}$ (see Definition 3.7). Thus, for every $n$-cosimplicial simplicial set $\boldsymbol{X}$ there is a natural isomorphism of sets

$$
\operatorname{SS}^{\boldsymbol{\Delta}^{n}}\left(\Delta^{(n)}, \boldsymbol{X}\right) \approx \mathrm{SS}^{\boldsymbol{\Delta}}(\Delta, \operatorname{diag} \boldsymbol{X})
$$

between the set of maps of $n$-cosimplicial simplicial sets $\Delta^{(n)} \rightarrow X$ and the set of maps of cosimplicial simplicial sets $\Delta \rightarrow \operatorname{diag} \boldsymbol{X}$ (see Definition 2.5).

2. The unit of the adjunction on the cosimplicial standard simplex $\Delta$ is the natural transformation $\alpha: \Delta \rightarrow \operatorname{diag} \Delta^{(n)}$ that on the object $[k]$ of $\boldsymbol{\Delta}$ is the diagonal map $\Delta[k] \rightarrow \Delta[k] \times \Delta[k] \times \cdots \times \Delta[k]$. Thus, the above isomorphism takes a map $f: \Delta^{(n)} \rightarrow \boldsymbol{X}$ in $\mathrm{SS}^{\Delta^{n}}$ to the composition $\Delta \stackrel{\alpha}{\rightarrow} \operatorname{diag} \Delta^{(n)} \stackrel{\operatorname{diag} f}{\longrightarrow} \operatorname{diag} \boldsymbol{X}$ in $\mathrm{SS}^{\boldsymbol{\Delta}}$ (see [2, Def. 3.7.1]). 
Proof Since the category of simplicial sets is cocomplete, the left Kan extension $\mathbf{L} \Delta$ of $\Delta$ exists and can be constructed pointwise (see [2, Thm. 3.7.2]). We view $\boldsymbol{\Delta}$ as the diagonal subcategory of $\boldsymbol{\Delta}^{n}$, and so for each object $\left(\left[p_{1}\right],\left[p_{2}\right], \ldots,\left[p_{n}\right]\right)$ of $\boldsymbol{\Delta}^{n}$, the simplicial set $\mathbf{L} \Delta\left(\left[p_{1}\right],\left[p_{2}\right], \ldots,\left[p_{n}\right]\right)$ is the colimit of the $\left(\boldsymbol{\Delta} \downarrow\left(\left[p_{1}\right],\left[p_{2}\right], \ldots,\left[p_{n}\right]\right)\right)$ diagram of simplicial sets that takes the object

$$
\left(\alpha_{1}, \alpha_{2}, \ldots, \alpha_{n}\right):([k],[k], \ldots,[k]) \longrightarrow\left(\left[p_{1}\right],\left[p_{2}\right], \ldots,\left[p_{n}\right]\right)
$$

of $\left(\boldsymbol{\Delta} \downarrow\left(\left[p_{1}\right],\left[p_{2}\right], \ldots,\left[p_{n}\right]\right)\right)$ to the standard $k$-simplex $\Delta[k]$. That object is the product for $1 \leq i \leq n$ of morphisms $\alpha_{i}:[k] \rightarrow\left[p_{i}\right]$ in $\Delta$, i.e., the product for $1 \leq i \leq n$ of a $k$-simplex of $\Delta\left[p_{i}\right]$, i.e., a $k$-simplex of $\Delta\left[p_{1}\right] \times \Delta\left[p_{2}\right] \times \cdots \times \Delta\left[p_{n}\right]$. Thus, $\mathbf{L} \Delta\left(\left[p_{1}\right],\left[p_{2}\right], \ldots,\left[p_{n}\right]\right)$ is the colimit of the diagram indexed by the category of simplices of $\Delta\left[p_{1}\right] \times \Delta\left[p_{2}\right] \times \cdots \times \Delta\left[p_{n}\right]$ (see Definition 3.9) that takes each $k$-simplex of $\Delta\left[p_{1}\right] \times \Delta\left[p_{2}\right] \times \cdots \times \Delta\left[p_{n}\right]$ to the standard $k$-simplex $\Delta[k]$, and so Lemma 3.10 implies that $\mathbf{L} \Delta\left(\left[p_{1}\right],\left[p_{2}\right], \ldots,\left[p_{n}\right]\right) \approx \Delta\left[p_{1}\right] \times \Delta\left[p_{2}\right] \times \cdots \times \Delta\left[p_{n}\right]$.

For the unit of the adjunction, note that a map of simplicial sets with domain $\Delta[k]$ is entirely determined by what it does to the nondegenerate $k$-simplex of $\Delta[k]$, which is the identity map of $[k]$. The natural transformation $\alpha: \Delta \rightarrow \operatorname{diag} \Delta^{(n)}$ on the object $[k]$ of $\Delta$ takes that nondegenerate $k$-simplex of $\Delta[k]$ to the $k$-simplex of $\Delta[k] \times \Delta[k] \times \cdots \times \Delta[k]$ that is the image under the diagonal embedding of the identity map of $[k]$, which is the map $[k] \rightarrow([k],[k], \ldots,[k])$ whose projection onto each factor is the identity map of $[k]$, i.e., the product of the nondegenerate $k$-simplices of each factor.

\subsection{Quillen functors, but not Quillen equivalences}

Let $\mathcal{M}$ be a model category. We show in Theorem 3.12 and Corollary 3.13 that the right Quillen functor diag: $\mathcal{M}^{\boldsymbol{\Delta}^{n}} \rightarrow \mathcal{M}^{\boldsymbol{\Delta}}$ (see Theorem 3.6) and its left adjoint $\mathbf{L}: \mathcal{M} \boldsymbol{N}^{\boldsymbol{\Delta}} \rightarrow$ $\mathcal{M}^{\Delta^{n}}$ are not Quillen equivalences when $\mathcal{M}$ is either the model category of simplicial sets or the model category of topological spaces (see [6, Def. 8.5.20]).

Theorem 3.12 If $\mathcal{M}=\mathrm{SS}$, the model category of simplicial sets, and $n \geq 2$, then the Quillen functor diag: $\mathcal{M}^{\Delta^{n}} \rightarrow \mathcal{M}^{\Delta}$ and its left adjoint $\mathbf{L}: \mathcal{M}^{\Delta} \rightarrow \mathcal{M}^{\overline{\Delta^{n}}}$ are not Quillen equivalences between the Reedy model categories $\mathcal{M}^{\Delta^{n}}$ and $\mathcal{M}{ }^{\Delta}$.

Proof We will discuss the case $n=2$; the other cases are similar. We will construct a cofibrant cosimplicial simplicial set $\boldsymbol{X}$, a fibrant 2-cosimplicial simplicial set $\mathrm{Y}$, and a weak equivalence $f: \boldsymbol{X} \rightarrow \operatorname{diag} \boldsymbol{Y}$ such that the corresponding map $\mathbf{L} \boldsymbol{X} \rightarrow \boldsymbol{Y}$ is not a weak equivalence.

For each $k \geq 0$, let $(\Delta[k])^{0}$ denote the 0 -skeleton of $\Delta[k]$, and let $\boldsymbol{X}$ be the cosimplicial simplicial set that is the degreewise 0 -skeleton of the cosimplicial standard simplex $\Delta$, so that $\boldsymbol{X}^{k}=(\Delta[k])^{0}$. Since the maximal augmentation of $\boldsymbol{X}$ is empty, $\boldsymbol{X}$ is cofibrant (see [6, Cor. 15.9.10]).

Let $\boldsymbol{W}$ be the 2-cosimplicial simplicial set obtained from $\boldsymbol{X}$ by making it constant in the second index, i.e., $\boldsymbol{W}^{(p, q)}=\boldsymbol{X}^{p}=(\Delta[p])^{0}$, and let $\boldsymbol{W} \rightarrow \boldsymbol{Y}$ be a fibrant approximation to $\boldsymbol{W}$, so that $\boldsymbol{W} \rightarrow \boldsymbol{Y}$ is a weak equivalence of 2-cosimplicial simplicial 
sets and $\boldsymbol{Y}$ is fibrant. There is an obvious isomorphism of cosimplicial simplicial sets $\boldsymbol{X} \rightarrow \operatorname{diag} \boldsymbol{W}$, and our map $f: \boldsymbol{X} \rightarrow \operatorname{diag} \boldsymbol{Y}$ is the composition $\boldsymbol{X} \rightarrow \operatorname{diag} \boldsymbol{W} \rightarrow$ $\operatorname{diag} \boldsymbol{Y}$; it is the composition of an isomorphism and an objectwise weak equivalence, and so it is an objectwise weak equivalence, i.e., a weak equivalence of cosimplicial simplicial sets.

The functor $\mathbf{L}: \mathrm{SS}^{\boldsymbol{\Delta}} \rightarrow \mathrm{SS}^{\boldsymbol{\Delta}^{2}}$ takes the cosimplicial standard simplex $\Delta$ to the 2 cosimplicial product of standard simplices $\Delta^{(2)}$ (see Theorem 3.11). Since the colimit of a diagram of simplicial sets is constructed degreewise, and every simplicial set in the diagram whose colimit is $(\mathbf{L} \boldsymbol{X})^{(p, q)}$ is discrete (i.e., has all face and degeneracy operators isomorphisms), each $(\mathbf{L} \boldsymbol{X})^{(p, q)}$ is also discrete, and so $\mathbf{L} \boldsymbol{X}=\left(\Delta^{(2)}\right)^{0}$, the degreewise 0 -skeleton of $\Delta^{(2)}$. Thus, $(\mathbf{L} \boldsymbol{X})^{(1,1)}$ is the 0 -skeleton of $\Delta[1] \times \Delta[1]$, and has four path components, while $\boldsymbol{Y}^{(1,1)}$ is weakly equivalent to $\boldsymbol{W}^{(1,1)}=(\Delta[1])^{0}$, and has two path components. Thus, the map $\mathbf{L} \boldsymbol{X} \rightarrow \boldsymbol{Y}$ is not a weak equivalence.

Corollary 3.13 If $\mathcal{M}=$ Top, the model category of topological spaces, and $n \geq 2$, then the Quillen functor diag: $\mathcal{M}^{\boldsymbol{\Delta}^{n}} \rightarrow \mathcal{M}^{\mathbf{\Delta}}$ and its left adjoint $\mathbf{L}: \mathcal{M}^{\mathbf{\Delta}} \rightarrow \mathcal{M}^{\boldsymbol{\Delta}^{n}}$ are not Quillen equivalences between the Reedy model categories $\mathcal{M}^{\Delta^{n}}$ and $\mathcal{M}{ }^{\Delta}$.

Proof The geometric realization of the example in Theorem 3.12 is a cofibrant cosimplicial space $\boldsymbol{X}$, a fibrant 2-cosimplicial space $\boldsymbol{Y}$, and a weak equivalence $f: \boldsymbol{X} \rightarrow$ $\operatorname{diag} \boldsymbol{Y}$ such that the corresponding map $\mathbf{L} \boldsymbol{X} \rightarrow \boldsymbol{Y}$ is not a weak equivalence.

\section{Proof of Proposition 3.3}

Since $\overleftarrow{\Delta^{n}}=\overleftarrow{\Delta} \times \overleftarrow{\Delta} \times \cdots \times \overleftarrow{\Delta}$ (see [6, Prop. 15.1.6]), the objects of the matching category $\partial\left(([k],[k], \ldots,[k]) \downarrow \overleftarrow{\Delta^{n}}\right)$ are the maps

$$
\left([k] \rightarrow\left[p_{1}\right],[k] \rightarrow\left[p_{2}\right], \ldots,[k] \rightarrow\left[p_{n}\right]\right)
$$

in $\Delta^{n}$ such that each $[k] \rightarrow\left[p_{i}\right]$ is a surjection and such that at least one of them is not the identity map. The diagonal embedding of $\Delta$ into $\Delta^{n}$ (see Definition 2.5) takes the matching category $\partial([k] \downarrow \overleftarrow{\Delta})$ to the full subcategory of $\partial\left(([k],[k], \ldots,[k]) \downarrow \overleftarrow{\Delta^{n}}\right)$ with objects the maps

$$
\left\{\phi^{n} \mid \phi:[k] \rightarrow[p] \text { is a surjection and is not the identity map }\right\}
$$

and we will identify $\partial([k] \downarrow \overleftarrow{\Delta})$ with its image in $\partial\left(([k],[k], \ldots,[k]) \downarrow \overleftarrow{\boldsymbol{\Delta}^{n}}\right)$. Our map

$$
\begin{aligned}
& P_{k}^{\boldsymbol{\Delta}^{n}}=\left(\lim _{\partial\left(([k],[k], \ldots,[k]) \downarrow \overleftarrow{\boldsymbol{\Delta}^{n}}\right)} \boldsymbol{X}\right) \times\left(\lim _{\partial\left(([k],[k], \ldots,[k]) \downarrow \overleftarrow{\left.\Delta^{n}\right)}\right.} \boldsymbol{Y}\right)^{\boldsymbol{Y}^{(k, k, \ldots, k)}} \\
& \longrightarrow \quad P_{k}^{\boldsymbol{\Delta}}=\left(\lim _{\partial([k] \downarrow \overleftarrow{\Delta})} \boldsymbol{X}\right) \times\left(\lim _{\partial([k] \downarrow \overleftarrow{\Delta})} \boldsymbol{Y}\right)^{\boldsymbol{Y}^{(k, k, \ldots, k)}}
\end{aligned}
$$


(see Definition 3.1) is induced by restricting the functors $\boldsymbol{X}$ and $\boldsymbol{Y}$ to this subcategory. We will define a nested sequence of full subcategories of $\partial\left(([k],[k], \ldots,[k]) \downarrow \overleftarrow{\Delta^{n}}\right)$

$$
\partial([k] \downarrow \overleftarrow{\Delta})=\mathcal{C}_{-1} \subset \mathcal{C}_{0} \subset \cdots \subset \mathcal{C}_{n k-1}=\partial\left(([k],[k], \ldots,[k]) \downarrow \overleftarrow{\Delta^{n}}\right)
$$

and for $-1 \leq i \leq n k-1$ we will let $P_{i}$ be the pullback

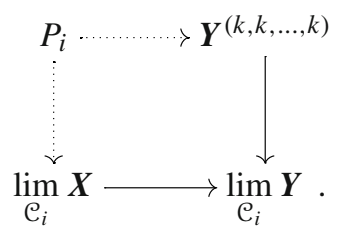

Thus, we will have a factorization of our map $P_{k}^{\Delta^{n}} \rightarrow P_{k}^{\Delta}$ as

$$
P_{k}^{\Delta^{n}}=P_{n k-1} \longrightarrow P_{n k-2} \longrightarrow \cdots \longrightarrow P_{-1}=P_{k}^{\Delta}
$$

and we will show that the map $P_{i+1} \rightarrow P_{i}$ is a fibration for $-1 \leq i \leq n k-2$.

Definition 4.1 If $n$ is a positive integer, $k$ is a nonnegative integer, and $-1 \leq i \leq$ $n k-1$, we let $\mathcal{C}_{i}$ be the full subcategory of $\partial\left(([k],[k], \ldots,[k]) \downarrow \overleftarrow{\Delta^{n}}\right)$ with objects the union of

- the objects of $\partial\left(([k],[k], \ldots,[k]) \downarrow \overleftarrow{\Delta^{n}}\right)$ whose target is of degree at most $i$, and - the objects of $\partial\left(([k],[k], \ldots,[k]) \downarrow \overleftarrow{\Delta^{n}}\right)$ in the image of the embedding of $\partial([k] \downarrow \overleftarrow{\Delta})$

That is, we let $\mathcal{C}_{i}$ be the full subcategory of $\partial\left(([k],[k], \ldots,[k]) \downarrow \overleftarrow{\Delta^{n}}\right)$ with objects the maps

$$
\left(\phi_{1}, \phi_{2}, \ldots, \phi_{n}\right):([k],[k], \ldots,[k]) \longrightarrow\left(\left[p_{1}\right],\left[p_{2}\right], \ldots,\left[p_{n}\right]\right)
$$

such that either $p_{1}+p_{2}+\cdots+p_{n} \leq i$ or $\phi_{1}=\phi_{2}=\cdots=\phi_{n}$. Note that, since every map in $\overleftarrow{\Delta}$ is an epimorphism, every map in $\partial\left(([k],[k], \ldots,[k]) \downarrow \overleftarrow{\Delta^{n}}\right)$ between objects in the image of the embedding of $\partial([k] \downarrow \overleftarrow{\Delta})$ must also be in the image of that embedding; thus, $\mathrm{C}_{-1}=\partial([k] \downarrow \overleftarrow{\Delta})$

Proposition 4.2 If $-1 \leq i \leq n k-2$, then the map $P_{i+1} \rightarrow P_{i}$ is a fibration.

Proof The objects of $\mathcal{C}_{i+1}$ that aren't in $\mathcal{C}_{i}$ are maps $\left([k] \rightarrow\left[p_{1}\right],[k] \rightarrow\right.$ $\left.\left[p_{2}\right], \ldots,[k] \rightarrow\left[p_{n}\right]\right)$ such that $p_{1}+p_{2}+\cdots+p_{n}=i+1$ (though not necessarily all such maps), and this set of maps can be divided into two subsets:

- the set $S_{i+1}$ of maps for which there exists an epimorphism $\psi:[k] \rightarrow[j]$ with $j<k$ and a factorization through $\psi^{n}:([k],[k], \ldots,[k]) \rightarrow([j],[j], \ldots,[j])$, 
- the set $T_{i+1}$ of maps for which there is no such factorization.

We let $\mathcal{C}_{i+1}^{\prime}$ be the full subcategory of $\partial\left(([k],[k], \ldots,[k]) \downarrow \overleftarrow{\Delta^{n}}\right)$ with objects the union of $S_{i+1}$ with the objects of $\mathcal{C}_{i}$, and define $P_{i+1}^{\prime}$ as the pullback

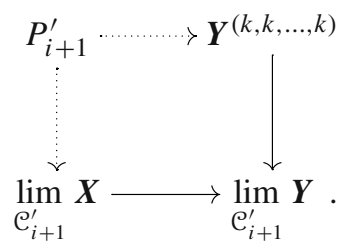

We have inclusions of categories $\mathcal{C}_{i} \subset \mathcal{C}_{i+1}^{\prime} \subset \mathcal{C}_{i+1}$, and the maps

$$
\lim _{\mathcal{C}_{i+1}} \boldsymbol{X} \longrightarrow \lim _{\mathcal{C}_{i}} \boldsymbol{X} \quad \text { and } \quad \lim _{\mathcal{C}_{i+1}} \boldsymbol{Y} \longrightarrow \lim _{\mathcal{C}_{i}} \boldsymbol{Y}
$$

factor as

$$
\lim _{\mathcal{C}_{i+1}} \boldsymbol{X} \longrightarrow \lim _{\mathcal{C}_{i+1}^{\prime}} \boldsymbol{X} \longrightarrow \lim _{\mathcal{C}_{i}} \boldsymbol{X} \quad \text { and } \quad \lim _{\mathcal{C}_{i+1}} \boldsymbol{Y} \longrightarrow \lim _{\mathcal{C}_{i+1}^{\prime}} \boldsymbol{Y} \longrightarrow \lim _{\mathcal{C}_{i}} \boldsymbol{Y}
$$

These factorizations induce a factorization

$$
P_{i+1} \longrightarrow P_{i+1}^{\prime} \longrightarrow P_{i} \quad \text { of the map } \quad P_{i+1} \longrightarrow P_{i}
$$

Proposition 4.3 asserts that the map $P_{i+1}^{\prime} \rightarrow P_{i}$ is an isomorphism and Proposition 4.4 asserts that the map $P_{i+1} \rightarrow P_{i+1}^{\prime}$ is a fibration.

Proposition 4.3 For $-1 \leq i \leq n k-2$, the map $P_{i+1}^{\prime} \rightarrow P_{i}$ is an isomorphism.

The proof of Proposition 4.3 is in Sect. 5 .

Proposition 4.4 For $-1 \leq i \leq n k-1$, the map $P_{i+1} \rightarrow P_{i+1}^{\prime}$ is a fibration.

The proof of Proposition 4.4 is in Sect. 6.

\section{Proof of Proposition 4.3}

Lemma 5.1 Every morphism

$$
\left(\alpha_{1}, \alpha_{2}, \ldots, \alpha_{n}\right):([k],[k], \ldots,[k]) \longrightarrow\left(\left[p_{1}\right],\left[p_{2}\right], \ldots,\left[p_{n}\right]\right)
$$

in $\overleftarrow{\Delta^{n}}$ with domain a diagonal object has a terminal factorization through a diagonal morphism, i.e., an epimorphism $\beta:[k] \rightarrow[q]$ and a factorization

$$
([k],[k], \ldots,[k]) \stackrel{\beta^{n}}{\longrightarrow}([q],[q], \ldots,[q]) \longrightarrow\left(\left[p_{1}\right],\left[p_{2}\right], \ldots,\left[p_{n}\right]\right)
$$


of $\left(\alpha_{1}, \alpha_{2}, \ldots, \alpha_{n}\right)$ such that every epimorphism $\gamma:[k] \rightarrow[r]$ and factorization

$$
([k],[k], \ldots,[k]) \stackrel{\gamma^{n}}{\longrightarrow}([r],[r], \ldots,[r]) \longrightarrow\left(\left[p_{1}\right],\left[p_{2}\right], \ldots,\left[p_{n}\right]\right)
$$

of $\left(\alpha_{1}, \alpha_{2}, \ldots, \alpha_{n}\right)$ through a diagonal morphism of $\boldsymbol{\Delta}^{n}$ factors uniquely as

$([k],[k], \ldots,[k]) \stackrel{\gamma^{n}}{\rightarrow}([r],[r], \ldots,[r]) \stackrel{\delta^{n}}{\rightarrow}([q],[q], \ldots,[q]) \longrightarrow\left(\left[p_{1}\right],\left[p_{2}\right], \ldots,\left[p_{n}\right]\right)$

with $\delta \gamma=\beta$, for $\delta:[r] \rightarrow[q]$ an epimorphism.

Proof Each of the epimorphisms $\alpha_{j}:[k] \rightarrow\left[p_{j}\right]$ is determined by the set $U_{j}$ of integers $i$ such that $\alpha_{j}(i)=\alpha_{j}(i+1)$. We let $U=\bigcap_{1 \leq j \leq n} U_{j}$. The set $U$ now determines an epimorphism $\beta:[k] \rightarrow[q]$ for some $q \leq k$, and the terminal factorization of $\alpha$ is the factorization through $\beta^{n}:([k],[k], \ldots,[k]) \rightarrow([q],[q], \ldots,[q])$.

Proposition 5.2 For $-1 \leq i \leq n k-2$, the inclusion of categories $\mathrm{C}_{i} \subset \mathrm{C}_{i+1}^{\prime}$ is left cofinal (see [6, Def. 14.2.1]).

Proof Let $\alpha=\left([k] \rightarrow\left[p_{1}\right],[k] \rightarrow\left[p_{2}\right], \ldots,[k] \rightarrow\left[p_{n}\right]\right)$ be an object of $\mathrm{C}_{i+1}^{\prime}$ that isn't in $\mathfrak{C}_{i}$. Since every morphism in $\overleftarrow{\Delta^{n}}$ lowers degree, the only objects of $\left(\mathfrak{C}_{i} \downarrow \alpha\right)$ are factorizations of $\alpha$ with intermediate object of degree greater than $i+1$, which must then be through $\phi^{n}:([k],[k], \ldots,[k]) \rightarrow([j],[j], \ldots,[j])$ for some epimorphism $\phi:[k] \rightarrow[j]$ with $j<k$. Now Lemma 5.1 implies that there is a terminal such factorization, i.e., one through which all other factorizations factor uniquely. That terminal factorization is a terminal object of the overcategory $\left(\mathfrak{C}_{i} \downarrow \alpha\right)$, and so that overcategory is nonempty and connected. Thus, the inclusion $\mathcal{C}_{i} \subset \mathcal{C}_{i+1}^{\prime}$ is left cofinal.

Proof of Proposition 4.3 For $-1 \leq i \leq n k-2$, Proposition 5.2 implies that the inclusion of categories $\mathcal{C}_{i} \subset \mathcal{C}_{i+1}^{\prime}$ is left cofinal, and so the maps $\lim _{\mathcal{C}_{i+1}^{\prime}} X \rightarrow \lim _{\mathcal{C}_{i}} X$ and $\lim _{\mathcal{C}_{i+1}^{\prime}} \boldsymbol{Y} \rightarrow \lim _{\mathcal{C}_{i}} \boldsymbol{Y}$ are isomorphisms (see [6, Thm. 14.2.5]). Thus, the induced map $P_{i+1}^{\prime} \rightarrow P_{i}$ is an isomorphism.

\section{Proof of Proposition 4.4}

Notation 6.1 To save space, we will use the following compact notation:

- The symbol $[\vec{p}]$ will denote an object $\left(\left[p_{1}\right],\left[p_{2}\right], \ldots,\left[p_{n}\right]\right)$ of $\boldsymbol{\Delta}^{n}$, and

- the symbol $[k]^{n} \rightarrow[\vec{p}]$ will denote an object $([k],[k], \ldots,[k]) \rightarrow\left(\left[p_{1}\right]\right.$, $\left.\left[p_{2}\right], \ldots,\left[p_{n}\right]\right)$ of the matching category $\partial\left(([k],[k], \ldots,[k]) \downarrow \overleftarrow{\Delta^{n}}\right)$ 
Lemma 6.2 For each $n$-cosimplicial object $\boldsymbol{X}$ in $\mathcal{M}$ there is a pullback square

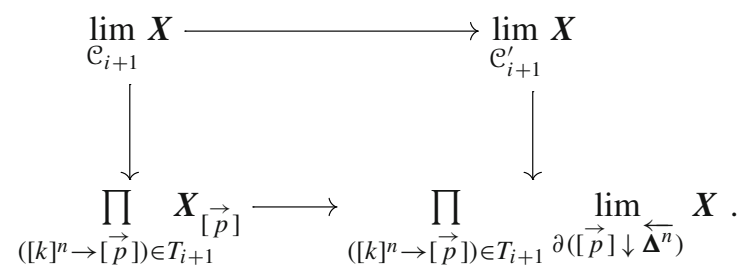

Proof For every element $[k]^{n} \rightarrow[\vec{p}]$ of $T_{i+1}$, an object of the matching category $\partial\left([\vec{p}] \downarrow \overleftarrow{\Delta^{n}}\right)$ is a map to an object of degree at most $i$, and so there is a functor $\partial\left([\vec{p}] \downarrow \overleftarrow{\Delta^{n}}\right) \rightarrow \mathcal{C}_{i+1}^{\prime}$ that takes the object $\left(\left[p_{1}\right],\left[p_{2}\right], \ldots,\left[p_{n}\right]\right) \rightarrow$ $\left(\left[q_{1}\right],\left[q_{2}\right], \ldots,\left[q_{n}\right]\right)$ to the composition

$$
([k],[k], \ldots,[k]) \rightarrow\left(\left[p_{1}\right],\left[p_{2}\right], \ldots,\left[p_{n}\right]\right) \rightarrow\left(\left[q_{1}\right],\left[q_{2}\right], \ldots,\left[q_{n}\right]\right)
$$

this induces the map $\lim _{\mathcal{C}_{i+1}^{\prime}} X \rightarrow \lim _{\partial\left([\vec{p}] \downarrow \overleftarrow{\Delta^{n}}\right)} \boldsymbol{X}$ that is the projection of the right hand vertical map onto the factor indexed by $[k]^{n} \rightarrow[\vec{p}]$. We thus have a commutative square as in Diagram 6.3.

The objects of $\mathcal{C}_{i+1}$ are the objects of $\mathcal{C}_{i+1}^{\prime}$ together with the elements of $T_{i+1}$, and so a map to $\lim _{\mathcal{C}_{i+1}} \boldsymbol{X}$ is determined by its compositions with the above horizontal map to $\lim _{\mathcal{C}_{i+1}^{\prime}} \boldsymbol{X}$ and with the above vertical map to $\prod_{\left([k]^{n} \rightarrow[\vec{p}]\right) \in T_{i+1}} \boldsymbol{X}_{[\vec{p}]}$. Since there are no non-identity morphisms in $\mathcal{C}_{i+1}$ with codomain an element of $T_{i+1}$, and the only non-identity morphisms with domain an element $[k]^{n} \rightarrow[\vec{p}]$ of $T_{i+1}$ are the objects of the matching category $\partial\left([\vec{p}] \downarrow \overleftarrow{\Delta^{n}}\right)$, maps to $\lim _{\mathcal{C}_{i+1}^{\prime}} X$ and to $\prod_{\left([k]^{n} \rightarrow[\vec{p}]\right) \in T_{i+1}} X_{[\vec{p}]}$ determine a map to $\lim _{\mathcal{C}_{i+1}} X$ if and only if their compositions to $\prod_{\left([k]^{n} \rightarrow[\vec{p}]\right) \in T_{i+1}} \lim _{\partial\left([\vec{p}] \downarrow \overleftarrow{\Delta^{n}}\right)} \boldsymbol{X}$ agree. Thus, the diagram is a pullback square.

Define $Q$ and $R$ by letting the squares
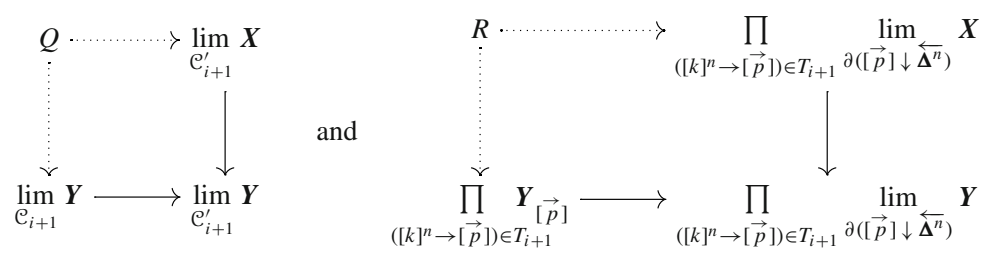
be pullbacks, and consider the commutative diagram

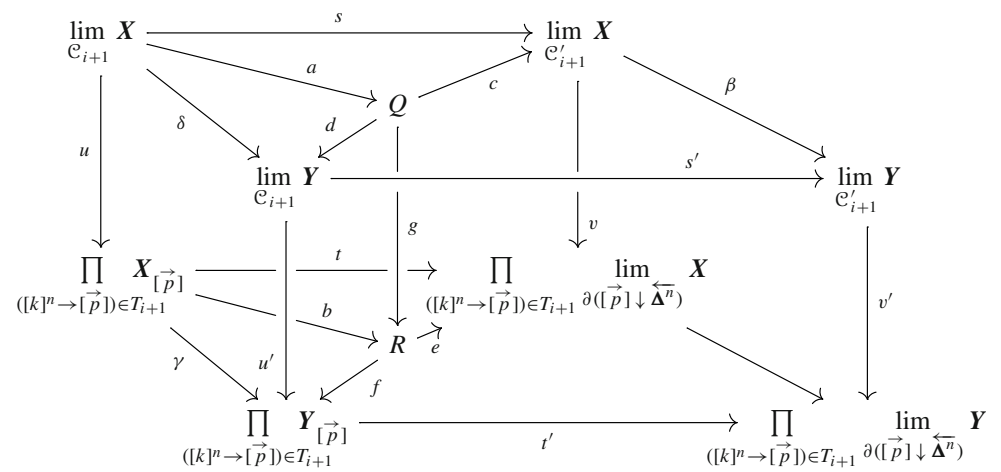

Lemma 6.2 implies that the front and back rectangles are pullbacks.

\section{Lemma 6.6 The square}

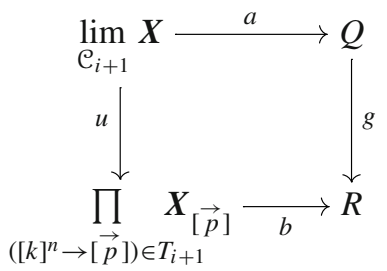

is a pullback.

Proof Let $W$ be an object of $\mathcal{M}$ and let $h: W \rightarrow \prod_{\left([k]^{n} \rightarrow[\vec{p}]\right) \in T_{i+1}} \boldsymbol{X}_{[\vec{p}]}$ and $k: W \rightarrow$ $Q$ be maps such that $g k=b h$; we will show that there is a unique map $\phi: W \rightarrow$ $\lim _{\mathcal{C}_{i+1}} \boldsymbol{X}$ such that $a \phi=k$ and $u \phi=h$.

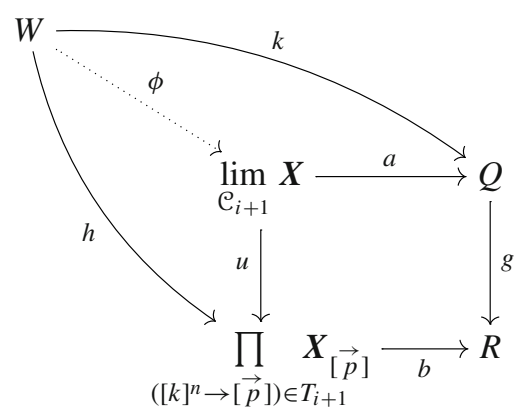

The map $c k: W \rightarrow \lim _{\mathcal{C}_{i+1}^{\prime}} \boldsymbol{X}$ has the property that $v(c k)=e g k=e b h=t h$, and since the back rectangle of Diagram 6.5 is a pullback, the maps $c k$ and $h$ induce a map $\phi: W \rightarrow \lim _{\mathcal{C}_{i+1}} X$ such that $u \phi=h$ and $s \phi=c k$. We must show that $a \phi=k$, and since $Q$ is a pullback as in Diagram 6.4, this is equivalent to showing that $c a \phi=c k$ and $d a \phi=d k$. 
Since $c k=s \phi=c a \phi$, we need only show that $d a \phi=d k$. Since the front rectangle of Diagram 6.5 is a pullback, it is sufficient to show that $s^{\prime} d a \phi=s^{\prime} d k$ and $u^{\prime} d a \phi=u^{\prime} d k$. For the first of those, we have

$$
s^{\prime} d a \phi=s^{\prime} \delta \phi=\beta s \phi=\beta c k=s^{\prime} d k
$$

and for the second, we have

$$
u^{\prime} d a \phi=u^{\prime} \delta \phi=\gamma u \phi=f b u \phi=f b h=f g k=u^{\prime} d k
$$

and so the map $\phi$ satisfies $a \phi=k$ and $u \phi=h$.

To see that $\phi$ is the unique such map, let $\psi: W \rightarrow \lim _{\mathcal{C}_{i+1}} \boldsymbol{X}$ be another map such that $a \psi=k$ and $u \psi=h$. We will show that $s \psi=s \phi$ and $u \psi=u \phi$; since the back rectangle of Diagram 6.5 is a pullback, this will imply that $\psi=\phi$.

Since $u \psi=h=u \phi$, we need only show that $s \psi=s \phi$, which follows because $s \psi=c a \psi=c k=s \phi$.

Lemma 6.8 If $\boldsymbol{X} \rightarrow \boldsymbol{Y}$ is a fibration of $n$-cosimplicial objects, then the natural map

$$
a: \lim _{\mathcal{C}_{i+1}} \boldsymbol{X} \longrightarrow Q=\lim _{\mathcal{C}_{i+1}^{\prime}} \boldsymbol{X} \times\left(\lim _{\mathcal{C}_{i+1}^{\prime}} \boldsymbol{Y}\right) \lim _{\mathcal{C}_{i+1}} \boldsymbol{Y}
$$

(see Diagrams 6.4 and 6.7) is a fibration.

Proof Lemma 6.6 gives us the pullback square in Diagram 6.7, where $Q$ and $R$ are defined by the pullbacks in Diagram 6.4. Since $\boldsymbol{X} \rightarrow \boldsymbol{Y}$ is a fibration of $n$-cosimplicial objects, the map $b: \prod_{\left([k]^{n} \rightarrow[\vec{p}]\right) \in T_{i+1}} \boldsymbol{X}_{[\vec{p}]} \rightarrow R$ is a product of Reedy relative matching maps which are assumed to be fibrations and is thus a fibration, and so the map $\lim _{\mathfrak{C}_{i+1}} \boldsymbol{X} \rightarrow Q=\lim _{\mathfrak{C}_{i+1}^{\prime}} \boldsymbol{X} \times_{\left(\lim _{\mathcal{C}_{i+1}^{\prime}} \boldsymbol{Y}\right)} \lim _{\mathfrak{C}_{i+1}} \boldsymbol{Y}$ is a pullback of a fibration and is thus a fibration.

Lemma 6.9 (Reedy) If both the front and back squares in the diagram

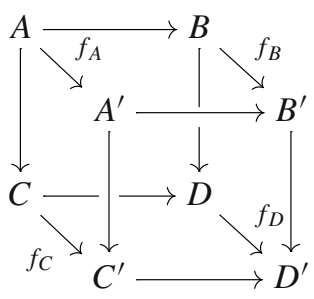

are pullbacks and both $f_{B}: B \rightarrow B^{\prime}$ and $C \rightarrow C^{\prime} \times_{D^{\prime}}$ D are fibrations, then $f_{A}: A \rightarrow$ $A^{\prime}$ is a fibration.

Proof This is the dual of a lemma of Reedy (see [6, Lem. 7.2.15 and Rem. 7.1.10]). 
Proof of Proposition 4.4 We have a commutative diagram

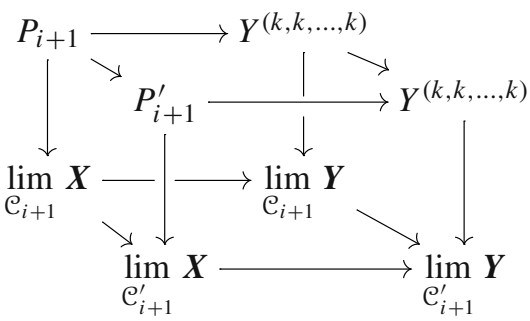

in which the front and back squares are pullbacks, and so Lemma 6.9 implies that it is sufficient to show that the map

$$
\lim _{\mathcal{C}_{i+1}} \boldsymbol{X} \longrightarrow \lim _{\mathcal{C}_{i+1}^{\prime}} \boldsymbol{X} \times{ }_{\left(\lim _{\mathcal{C}_{i+1}^{\prime}} \boldsymbol{Y}\right)} \lim _{\mathcal{C}_{i+1}} \boldsymbol{Y}
$$

is a fibration; that is the statement of Lemma 6.8 .

\section{Total objects and homotopy limits}

The remainder of this article will closely follow the notation and viewpoint of [6] on simplicial frames. The definitions of homotopy limits and total objects for (possibly non-simplicial) model categories depend on the definitions of simplicial frames (see [6, Sec. 16.6]) and Reedy simplicial frames (see [6, Sec. 16.7]). In addition to their definitions, the important points are

- the existence (see [6, Thm. 16.6.9 and Thm. 16.7.11]) and essential uniqueness (see [6, Thm. 16.6.10, Thm. 16.7.6, and Thm. 16.7.14]) of simplicial frames and Reedy simplicial frames, and

- the agreement between homotopy cotensors and the actual cotensor when using the standard simplicial frame on a simplicial model category (see [6, Prop. 16.6.6]).

We show that the Bousfield-Kan map from the total object of a multicosimplicial object to its homotopy limit is a weak equivalence for Reedy fibrant multicosimplicial objects. We also show that this behaves well with respect to passing to the diagonal cosimplicial object of a multicosimplicial object.

\subsection{The total object of a multicosimplicial object}

Definition 7.1 If $n$ is a positive integer, $\mathcal{M}$ is a model category, $\boldsymbol{X}$ is an $n$-cosimplicial object in $\mathcal{M}$, and $\widehat{\boldsymbol{X}}$ is a Reedy simplicial frame on $\boldsymbol{X}$ (see [6, Def. 16.7.8]), then the total object Tot $\boldsymbol{X}$ of $\boldsymbol{X}$ is the object of $\mathcal{M}$ that is the end (see [6, Def. 18.3.2], [7, pp. 218-223], or [3, p. 329]) of the functor $\widehat{\boldsymbol{X}}^{\Delta^{(n)}}: \boldsymbol{\Delta}^{n} \times\left(\boldsymbol{\Delta}^{n}\right)^{\text {op }} \rightarrow \mathcal{M}$. This is a subobject of the product 


$$
\prod_{k_{1} \geq 0, k_{2} \geq 0, \ldots, k_{n} \geq 0}\left(\widehat{\boldsymbol{X}}^{\left(k_{1}, k_{2}, \ldots, k_{n}\right)}\right)^{\left(\Delta\left[k_{1}\right] \times \Delta\left[k_{2}\right] \times \cdots \times \Delta\left[k_{n}\right]\right)}
$$

and is denoted hom $\widehat{\Delta}_{\widehat{\boldsymbol{X}}}^{n}\left(\Delta^{(n)}, \boldsymbol{X}\right)$ in [6, Def. 19.2.2] and

$$
\int_{\left(\left[k_{1}\right],\left[k_{2}\right], \ldots,\left[k_{n}\right]\right)}\left(\widehat{\boldsymbol{X}}^{\left(k_{1}, k_{2}, \ldots, k_{n}\right)}\right)^{\left(\Delta\left[k_{1}\right] \times \Delta\left[k_{2}\right] \times \cdots \times \Delta\left[k_{n}\right]\right)}
$$

in [7, pp. 218-223].

Example 7.2 If $n$ is a positive integer, $\mathcal{M}$ is the category of simplicial sets, $\boldsymbol{X}$ is an $n$-cosimplicial object in $\mathcal{M}$, and $\widehat{\boldsymbol{X}}$ is the standard simplicial frame on $\boldsymbol{X}$, then Tot $\boldsymbol{X}$ is the simplicial set of maps of $n$-cosimplicial simplicial sets from $\Delta^{(n)}$ to $\boldsymbol{X}$, i.e., a subset of the product simplicial set $\prod_{\left(\left[k_{1}\right],\left[k_{2}\right], \ldots,\left[k_{n}\right]\right)}\left(\boldsymbol{X}^{\left(k_{1}, k_{2}, \ldots, k_{n}\right)}\right)^{\left(\Delta\left[k_{1}\right] \times \Delta\left[k_{2}\right] \times \cdots \times \Delta\left[k_{n}\right]\right)}$ (see [6, Prop. 16.6.6]).

If $n$ is a positive integer, $\mathcal{M}$ is the category of topological spaces, $\boldsymbol{X}$ is an $n$ cosimplicial object in $\mathcal{M}$, and $\widehat{\boldsymbol{X}}$ is the standard simplicial frame on $\boldsymbol{X}$, then Tot $\boldsymbol{X}$ is the topological space of maps of $n$-cosimplicial spaces from $\left|\Delta^{(n)}\right|$ to $\boldsymbol{X}$, i.e., a subspace of the product space $\prod_{\left(\left[k_{1}\right],\left[k_{2}\right], \ldots,\left[k_{n}\right]\right)}\left(\boldsymbol{X}^{\left(k_{1}, k_{2}, \ldots, k_{n}\right)}\right)^{\left|\left(\Delta\left[k_{1}\right] \times \Delta\left[k_{2}\right] \times \cdots \times \Delta\left[k_{n}\right]\right)\right|}$.

Proposition 7.3 Ifn is a positive integer, $\mathcal{M}$ is a model category, $\boldsymbol{X}$ is an $n$-cosimplicial object in $\mathcal{M}$, and $\widehat{\boldsymbol{X}}$ is a Reedy simplicial frame on $\boldsymbol{X}$, then $\operatorname{diag} \widehat{\boldsymbol{X}}$ is a Reedy simplicial frame on $\operatorname{diag} \boldsymbol{X}$.

Proof Since a Reedy simplicial frame on a Reedy fibrant $n$-cosimplicial object is a Reedy fibrant diagram in $\left(\mathcal{M}^{\Delta^{n}}\right)^{\boldsymbol{\Delta}^{\mathrm{op}}} \approx\left(\mathcal{M}^{\boldsymbol{\Delta}^{\mathrm{op}}}\right)^{\boldsymbol{\Delta}^{n}}$, and the different Reedy model category structures on that category coincide (see [6, Thm. 15.5.2]), this follows from Corollary 3.5 .

Lemma 7.4 If $n$ is a positive integer, $\mathcal{M}$ is a model category, $\boldsymbol{X}$ is an $n$-cosimplicial object in $\mathcal{M}, \widehat{\boldsymbol{X}}$ is a simplicial frame on $\boldsymbol{X}$, and $W$ is an object of $\mathcal{M}$, then the cosimplicial simplicial set $\operatorname{diag} \mathcal{M}(W, \widehat{\boldsymbol{X}})$ equals the cosimplicial simplicial set $\mathcal{M}(W, \operatorname{diag} \widehat{\boldsymbol{X}})$.

Proof The $n$-cosimplicial simplicial set $\mathcal{M}(W, \widehat{\boldsymbol{X}})$ on $\left(\left[k_{1}\right],\left[k_{2}\right], \ldots,\left[k_{n}\right]\right)$ is the simplicial set $\mathcal{M}\left(W, \widehat{\boldsymbol{X}}^{\left(\left[k_{1}\right],\left[k_{2}\right], \ldots,\left[k_{n}\right]\right)}\right)$, and $\operatorname{diag} \mathcal{M}(W, \widehat{\boldsymbol{X}})$ is the cosimplicial simplicial set that is the restriction of that to the diagonal subcategory $\boldsymbol{\Delta}$ of $\boldsymbol{\Delta}^{n}$. The cosimplicial simplicial set $\mathcal{M}(W, \operatorname{diag} \widehat{\boldsymbol{X}})$ in degree $k$ is the simplicial set $\mathcal{M}\left(W, \widehat{\boldsymbol{X}}^{[k],[k], \ldots,[k]}\right)$.

Theorem 7.5 If $n$ is a positive integer, $\mathcal{M}$ is a model category, $\boldsymbol{X}$ is an $n$-cosimplicial object in $\mathcal{M}$, and $\widehat{\boldsymbol{X}}$ is a Reedy simplicial frame on $\boldsymbol{X}$, then diag $\widehat{\boldsymbol{X}}$ is a Reedy simplicial frame on $\operatorname{diag} \boldsymbol{X}$ (see Proposition 7.3) (which, by abuse of notation, we will also denote by $\widehat{\boldsymbol{X}}$ ) and there is a natural isomorphism

$$
\text { Tot } \boldsymbol{X} \approx \operatorname{Tot}(\operatorname{diag} \boldsymbol{X})
$$

from the total object of $\boldsymbol{X}$ to the total object of the diagonal cosimplicial object of $\boldsymbol{X}$. 
Proof For every object $W$ of $\mathcal{M}$ there are natural isomorphisms of sets

$$
\begin{array}{rlrl}
\mathcal{M}(W, \operatorname{Tot} \boldsymbol{X}) & =\mathcal{M}\left(W, \operatorname{hom}_{\widehat{\boldsymbol{X}}}^{\Delta^{n}}\left(\Delta^{(n)}, \boldsymbol{X}\right)\right) & \\
& \approx \operatorname{SS}^{\Delta^{n}}\left(\Delta^{(n)}, \mathcal{M}(W, \widehat{\boldsymbol{X}})\right) & & (\text { see [6, Prop. 19.2.13]) } \\
& \approx \operatorname{SS}^{\Delta}(\Delta, \operatorname{diag} \mathcal{M}(W, \widehat{\boldsymbol{X}})) & & (\text { see Theorem 3.11) } \\
& =\operatorname{SS}^{\Delta}(\Delta, \mathcal{M}(W, \operatorname{diag} \widehat{\boldsymbol{X}})) & & (\text { see Lemma 7.4) } \\
& \approx \mathcal{M}\left(W, \operatorname{hom} \widehat{\widehat{\boldsymbol{X}}}^{\Delta}(\Delta, \operatorname{diag} \boldsymbol{X})\right) & & \text { (see [6, Prop. 19.2.13)]) } \\
& =\mathcal{M}(W, \operatorname{Tot}(\operatorname{diag} \boldsymbol{X})) & &
\end{array}
$$

and the Yoneda lemma implies that the composition of those is induced by a natural isomorphism Tot $\boldsymbol{X} \approx \operatorname{Tot}(\operatorname{diag} \boldsymbol{X})$.

\subsection{Cosimplicial objects}

Definition 7.6 The Bousfield-Kan map $\phi: \mathrm{B}(\boldsymbol{\downarrow} \downarrow-) \rightarrow \Delta$ is the map of cosimplicial simplicial sets that for $k \geq 0$ and $n \geq 0$ takes the $n$-simplex

$$
\left(\left(\left[i_{0}\right] \stackrel{\sigma_{0}}{\longrightarrow}\left[i_{1}\right] \stackrel{\sigma_{1}}{\rightarrow} \cdots \stackrel{\sigma_{n-1}}{\longrightarrow}\left[i_{n}\right]\right), \tau:\left[i_{n}\right] \rightarrow[k]\right)
$$

of $\mathrm{B}(\boldsymbol{\Delta} \downarrow[k])$ to the $n$-simplex

$$
\left[\tau \sigma_{n-1} \sigma_{n-2} \cdots \sigma_{0}\left(i_{0}\right), \tau \sigma_{n-1} \sigma_{n-2} \cdots \sigma_{1}\left(i_{1}\right), \ldots, \tau \sigma_{n-1}\left(i_{n-1}\right), \tau\left(i_{n}\right)\right]
$$

of $\Delta[k]$ (see [6, Def. 18.7.1]). It is a weak equivalence of Reedy cofibrant cosimplicial simplicial sets (see [6, Prop. 18.7.2]).

Theorem 7.7 If $\mathcal{M}$ is a simplicial model category, $\boldsymbol{X}$ is a Reedy fibrant cosimplicial object in $\mathcal{M}$, and $\widehat{\boldsymbol{X}}$ is a Reedy simplicial frame on $\boldsymbol{X}$, then the map

$$
\operatorname{Tot} \boldsymbol{X}=\operatorname{hom}_{\widehat{\boldsymbol{X}}}^{\boldsymbol{\Delta}}(\Delta, \boldsymbol{X}) \stackrel{\operatorname{hom}_{\widehat{\boldsymbol{X}}}^{\boldsymbol{\Delta}}\left(\phi, 1_{X}\right)}{\longrightarrow} \operatorname{hom} \widehat{\widehat{\boldsymbol{X}}}_{\widehat{\widehat{C}}}^{\boldsymbol{\Delta}}(\mathrm{B}(\boldsymbol{\Delta} \downarrow-), \boldsymbol{X})=\operatorname{holim} \boldsymbol{X}
$$

(where $\phi: \mathrm{B}(\boldsymbol{\Delta} \downarrow-) \rightarrow \Delta$ is the Bousfield-Kan map of cosimplicial simplicial sets; see Definition 7.6) is a natural weak equivalence of fibrant objects $\operatorname{Tot} \boldsymbol{X} \cong \operatorname{holim} \boldsymbol{X}$, which we will also call the Bousfield-Kan map.

Proof Since the Bousfield-Kan map of cosimplicial simplicial sets is a weak equivalence of Reedy cofibrant cosimplicial simplicial sets, this follows from [6, Cor. 19.7.5].

\subsection{Multicosimplicial objects}

Lemma 7.8 Let $n$ be a positive integer, let $\mathcal{C}_{i}$ be a small category for $1 \leq i \leq n$, and let $\mathrm{C}=\prod_{1 \leq i \leq n} \mathrm{C}_{i}$. If $\alpha=\left(\alpha_{1}, \alpha_{2}, \ldots, \alpha_{n}\right)$ is an object of $\mathcal{C}$, then the 
overcategory $\left(\mathrm{C}_{\downarrow} \alpha\right) \approx \prod_{1 \leq i \leq n}\left(\complement_{i} \downarrow \alpha_{i}\right)$, and its classifying space (or nerve) is $\mathrm{B}(\mathcal{\complement} \downarrow \alpha) \approx \prod_{1 \leq i \leq n} \mathrm{~B}\left(\mathcal{C}_{i} \downarrow \alpha_{i}\right)$.

Proof This follows directly from the definitions.

Definition 7.9 Let $n$ be a positive integer. The product Bousfield-Kan map of $n$ cosimplicial simplicial sets $\phi^{n}: \mathrm{B}\left(\Delta^{n} \downarrow-\right) \rightarrow \Delta^{(n)}$ is the composition

$$
\mathrm{B}\left(\boldsymbol{\Delta}^{n} \downarrow-\right) \approx \prod_{1 \leq i \leq n} \mathrm{~B}(\boldsymbol{\Delta} \downarrow-) \stackrel{\phi^{n}}{\longrightarrow} \Delta^{(n)}
$$

(see Lemma 7.8) where $\phi$ is the Bousfield-Kan map of cosimplicial simplicial sets (see Definition 7.6).

Theorem 7.10 If $n$ is a positive integer, $\mathcal{M}$ is a simplicial model category, $\boldsymbol{X}$ is a Reedy fibrant $n$-cosimplicial object in $\mathcal{M}$, and $\widehat{\boldsymbol{X}}$ is a Reedy simplicial frame on $\boldsymbol{X}$, then the map

$$
\operatorname{Tot} \boldsymbol{X} \approx \operatorname{hom}_{\widehat{\boldsymbol{X}}}^{\mathbf{\Delta}^{n}}\left(\Delta^{(n)}, \boldsymbol{X}\right) \stackrel{\operatorname{hom}_{\widehat{\boldsymbol{X}}}^{\Delta^{n}}\left(\phi^{n}, 1_{\boldsymbol{X}}\right)}{\longrightarrow} \operatorname{hom}_{\widehat{\boldsymbol{X}}}^{\Delta^{n}}\left(\mathrm{~B}\left(\boldsymbol{\Delta}^{n} \downarrow-\right), \boldsymbol{X}\right) \approx \operatorname{holim} \boldsymbol{X}
$$

(where $\phi^{n}: \mathrm{B}\left(\boldsymbol{\Delta}^{n} \downarrow-\right) \rightarrow \Delta^{(n)}$ is the product Bousfield-Kan map of $n$-cosimplicial simplicial sets; see Definition 7.9) is a natural weak equivalence of fibrant objects Tot $\boldsymbol{X} \cong$ holim $\boldsymbol{X}$, which we will also call the product Bousfield-Kan map.

Proof Both $\mathrm{B}\left(\boldsymbol{\Delta}^{n} \downarrow-\right)$ and $\Delta^{(n)}$ are Reedy cofibrant $n$-cosimplicial simplicial sets (see [6, Cor. 15.6.7] and Lemma 3.8) that are contractible at every object of $\boldsymbol{\Delta}^{n}$. Thus, the product Bousfield-Kan map of $n$-cosimplicial simplicial sets is a weak equivalence of Reedy cofibrant $n$-cosimplicial sets, and so this follows from [6, Cor. 19.7.5].

\subsection{The homotopy limit and total object of the diagonal}

We first show that for an objectwise fibrant multicosimplicial object the canonical map from the homotopy limit to the homotopy limit of the diagonal cosimplicial object is a weak equivalence, and then we show that all the maps we've defined between the homotopy limits and total objects of a multicosimplicial object and its diagonal cosimplicial object commute.

Proposition 7.11 If $n$ is a positive integer, then the diagonal embedding $D: \Delta \rightarrow \Delta^{n}$ (see Definition 2.5) is homotopy left cofinal (see [6, Def. 19.6.1]).

Proof For an object $\left(\left[p_{1}\right],\left[p_{2}\right], \ldots,\left[p_{n}\right]\right)$ of $\boldsymbol{\Delta}^{n}$, an object of $\left(\boldsymbol{\Delta} \downarrow\left(\left[p_{1}\right],\left[p_{2}\right], \ldots\right.\right.$, $\left.\left.\left[p_{n}\right]\right)\right)$ is a map

$$
\left(\alpha_{1}, \alpha_{2}, \ldots, \alpha_{n}\right):([k],[k], \ldots,[k]) \longrightarrow\left(\left[p_{1}\right],\left[p_{2}\right], \ldots,\left[p_{n}\right]\right)
$$


in $\boldsymbol{\Delta}^{n}$, where each $\alpha_{i}:[k] \rightarrow\left[p_{i}\right]$ is a map in $\boldsymbol{\Delta}$, i.e., a $k$-simplex of $\Delta\left[p_{i}\right]$. Thus, that object is a $k$-simplex of $\Delta\left[p_{1}\right] \times \Delta\left[p_{2}\right] \times \cdots \times \Delta\left[p_{n}\right]$, and the category $\left(\Delta \downarrow\left(\left[p_{1}\right],\left[p_{2}\right], \ldots,\left[p_{n}\right]\right)\right)$ is the category of simplices of $\Delta\left[p_{1}\right] \times \Delta\left[p_{2}\right] \times$ $\cdots \times \Delta\left[p_{n}\right]$ (see Definition 3.9). Since the nerve of the category of simplices of a simplicial set is weakly equivalent to that simplicial set (see [6, Thm. 18.9.3]), $\mathrm{B}\left(\boldsymbol{\Delta} \downarrow\left(\left[p_{1}\right],\left[p_{2}\right], \ldots,\left[p_{n}\right]\right)\right)$ is weakly equivalent to $\Delta\left[p_{1}\right] \times \Delta\left[p_{2}\right] \times \cdots \times \Delta\left[p_{n}\right]$, and is thus contractible.

Theorem 7.12 If $n$ is a positive integer, $\mathcal{M}$ is a model category, and $\boldsymbol{X}$ is an objectwise fibrant $n$-cosimplicial object in $\mathcal{N}$, then the natural map holim $\boldsymbol{\Delta}^{n} \boldsymbol{X} \rightarrow \operatorname{holim}_{\boldsymbol{\Delta}} \operatorname{diag} \boldsymbol{X}$ induced by the diagonal embedding $D: \Delta \rightarrow \Delta^{n}$ is a weak equivalence.

Proof This follows from Proposition 7.11 and [6, Thm. 19.6.7].

Theorem 7.13 If $n$ is a positive integer, $\mathcal{M}$ is a model category, $\boldsymbol{X}$ is an $n$-cosimplicial object in $\mathcal{M}$, and $\widehat{\boldsymbol{X}}$ is a Reedy simplicial frame on $\boldsymbol{X}$, then the diagram

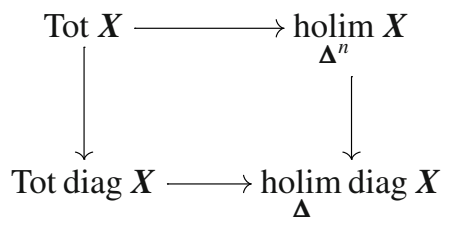

(where the upper horizontal map is the product Bousfield-Kan map (see Theorem 7.10), the lower horizontal map is the Bousfield-Kan map (see Theorem 7.7), the left vertical map is the isomorphism of Theorem 7.5, and the right vertical map is the natural map induced by the diagonal embedding $D: \Delta \rightarrow \Delta^{n}$ (see [6, Prop. 19.1.8])) commutes.

If $\boldsymbol{X}$ is objectwise fibrant, then the vertical maps in that diagram are weak equivalences. If $\boldsymbol{X}$ is Reedy fibrant, then all of the maps in that diagram are weak equivalences.

Proof To show that the diagram commutes, it is sufficient to show that if $W$ is an object of $\mathcal{M}$, then the diagram

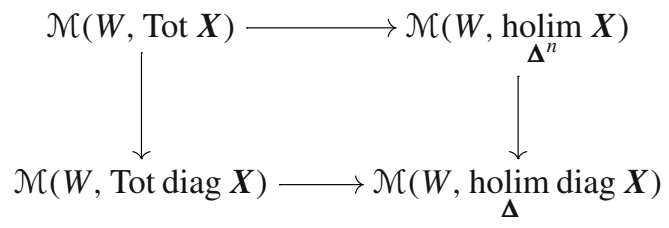


commutes. From [6, Prop. 19.2.13] we have natural isomorphisms

$$
\begin{aligned}
\mathcal{M}(W, \operatorname{Tot} \boldsymbol{X}) & =\mathcal{M}\left(W, \operatorname{hom}_{\widehat{\boldsymbol{X}}}^{\mathbf{\Delta}^{n}}\left(\Delta^{(n)}, \boldsymbol{X}\right)\right) \\
& \approx \mathrm{SS}^{\boldsymbol{\Delta}^{n}}\left(\Delta^{(n)}, \mathcal{M}(W, \widehat{\boldsymbol{X}})\right) \\
\mathcal{M}(W, \operatorname{holim} \boldsymbol{X}) & =\mathcal{M}\left(W, \operatorname{hom}_{\widehat{\boldsymbol{X}}}^{\boldsymbol{\Delta}^{n}}\left(\mathrm{~B}\left(\boldsymbol{\Delta}^{n} \downarrow-\right), \boldsymbol{X}\right)\right) \\
& \approx \mathrm{SS}^{\boldsymbol{\Delta}^{n}}\left(\mathrm{~B}\left(\boldsymbol{\Delta}^{n} \downarrow-\right), \mathcal{M}(W, \widehat{\boldsymbol{X}})\right) \\
\mathcal{M}(W, \operatorname{Tot} \operatorname{diag} \boldsymbol{X}) & =\mathcal{M}\left(W, \operatorname{hom}_{\widehat{\boldsymbol{X}}}^{\Delta}(\Delta, \operatorname{diag} \boldsymbol{X})\right) \\
& \approx \operatorname{SS}^{\boldsymbol{\Delta}}(\Delta, \mathcal{M}(W, \operatorname{diag} \widehat{\boldsymbol{X}})) \\
\mathcal{M}(W, \operatorname{holim} \operatorname{diag} \boldsymbol{X}) & =\mathcal{M}\left(W, \operatorname{hom} \widehat{\boldsymbol{X}}_{\widehat{\boldsymbol{X}}}^{\boldsymbol{\Delta}}(\mathrm{B}(\boldsymbol{\Delta} \downarrow-), \operatorname{diag} \boldsymbol{X})\right) \\
& \approx \operatorname{SS}^{\boldsymbol{\Delta}}(\mathrm{B}(\boldsymbol{\Delta} \downarrow-), \mathcal{M}(W, \operatorname{diag} \widehat{\boldsymbol{X}}))
\end{aligned}
$$

and so this is equivalent to showing that the diagram

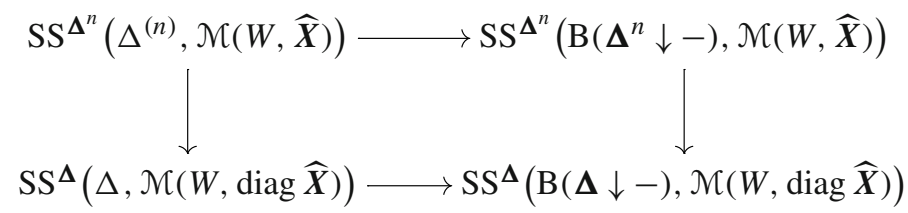

commutes. If $f \in \mathrm{SS}^{\boldsymbol{\Delta}^{n}}\left(\Delta^{(n)}, \mathcal{M}(W, \widehat{\boldsymbol{X}})\right)$, then the image of $f$ under the counterclockwise composition is the composition of

$$
\mathrm{B}(\boldsymbol{\Delta} \downarrow-) \stackrel{\phi}{\longrightarrow} \Delta \stackrel{\alpha}{\longrightarrow} \operatorname{diag}\left(\Delta^{(n)}\right) \stackrel{\operatorname{diag} f}{\longrightarrow} \operatorname{diag} \boldsymbol{X}
$$

(where $\phi$ is the Bousfield-Kan map of Definition 7.6 and $\alpha$ is as in Theorem 3.11) and the image of $f$ under the clockwise composition is

$$
\mathrm{B}(\boldsymbol{\Delta} \downarrow-) \stackrel{D_{*}}{\longrightarrow} \operatorname{diag}\left(\mathrm{B}\left(\boldsymbol{\Delta}^{n} \downarrow-\right)\right) \stackrel{\operatorname{diag} \phi^{n}}{\longrightarrow} \operatorname{diag}\left(\Delta^{(n)}\right) \stackrel{\operatorname{diag} f}{\longrightarrow} \operatorname{diag} \boldsymbol{X}
$$

(where $D_{*}$ is the map induced by the diagonal embedding $D: \Delta \rightarrow \boldsymbol{\Delta}^{n}$ and $\phi^{n}$ is the product Bousfield-Kan map of Definition 7.9). Since the diagram

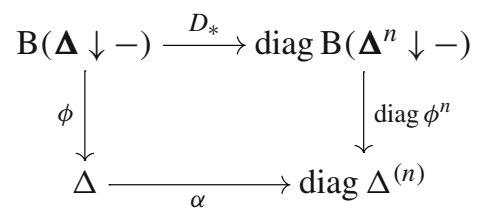

commutes, these two compositions are equal, and so our diagram commutes. 
Theorem 7.5 implies that the left vertical map is an isomorphism, and so it is a weak equivalence. If $\boldsymbol{X}$ is objectwise fibrant, then Theorem 7.12 implies that the right vertical map is a weak equivalence.

If $\boldsymbol{X}$ is Reedy fibrant, then Corollary 3.5 implies that $\operatorname{diag} \boldsymbol{X}$ is also Reedy fibrant and Proposition 7.3 implies that $\operatorname{diag} \widehat{\boldsymbol{X}}$ is a Reedy frame on $\operatorname{diag} \boldsymbol{X}$. Thus, Theorem 7.10 implies that the two horizontal maps are weak equivalences.

\section{References}

1. Bauer, K., Eldred, R., Johnson, B., McCarthy, R.: Combinatorial models for Taylor polynomials of functors (2015). http://arxiv.org/abs/1506.02112

2. Borceux, F.: Handbook of Categorical Algebra. 1. Encyclopedia of Mathematics and its Applications, vol. 50, p. xvi+345. Basic Category Theory. Cambridge University Press, Cambridge (1994)

3. Borceux, F.: Handbook of Categorical Algebra. 2. Encyclopedia of Mathematics and its Applications, vol. 51, p. xviii+443. Categories and Structures. Cambridge University Press, Cambridge (1994)

4. Bousfield, A.K., Kan, D.M.: Homotopy Limits, Completions and Localizations. Lecture Notes in Mathematics, vol. 304, p. v+348. Springer, Berlin (1972)

5. Dwyer, W., Miller, H., Neisendorfer, J.: Fibrewise completion and unstable Adams spectral sequences. Isr. J. Math. 66(1-3), 160-178 (1989)

6. Hirschhorn, P.S.: Model Categories and their Localizations, Mathematical Surveys and Monographs, p. xvi+457. American Mathematical Society, Providence, RI (2003)

7. MacLane, S.: Categories for the Working Mathematician. Graduate Texts in Mathematics, vol. 5, p. ix +262 . Springer, New York (1971)

8. Schubert, H.: Categories, Translated from the German by Eva Gray, p. xi+385. Springer, New York (1972)

9. Shipley, B.E.: Convergence of the homology spectral sequence of a cosimplicial space. Am. J. Math. 118(1), 179-207 (1996) 